consultant responsibility to diagnose dying and/or withdraw treatments. Both juniors and seniors described being less inclined to diagnose dying if the patient is young and/or has young children.

Conclusions This study has revealed challenges in recognition of dying perceived by Scottish doctors. The findings suggest this area of patient care is complex and uncertain, even for experienced practitioners. Comparing perceptions of senior and junior doctors gives insights for potential means of improved medical education. Recommendations include clearly defining the responsibilities of junior and senior team members, being explicit with learners about the often uncertain nature of recognising dying, and improved organisational factors to facilitate continuity of care.

\section{NEGATIVE PERSONAL EMOTIONAL IMPACT OF CARING FOR THE DYING - EXPECTATIONS OF FUTURE DOCTORS. A MULTICENTRE STUDY}

Pia Thiemann, Thelma Quince, Matthew Barclay, John Benson, James Brimicombe, Diana Wood, Stephen Barclay. Primary Care Unit, Department of Public Health and Primary Care, University of Cambridge, Cambridge, UK

\subsection{6/bmjspcare-2018-ASPabstracts.20}

Background End of life care (EOLC) is a large part of junior doctors' workload. Negative attitudes may impact on care provided and may be shaped during undergraduate medical training.

Aims We investigated:

- whether medical students expect EOLC to have a negative emotional impact on themselves

- demographic and psychological factors associated with such expectations.

Methods Multicentre cross-sectional online study of 1520 first and 954 final year medical students from 18 universities, (16 UK, 1 New Zealand, 1 Ireland). We assessed attitudes towards EOLC (Sullivan's statements), age, gender, course year, course type (standard or graduate), spirituality and experience of bereavement as well as psychological variables: death anxiety (Collett Lester Fear of Death Scale, COLFD), empathy (Davis's Interpersonal Reactivity Index, IRI) and depression (Hospital Depression Scale HADS-D). Factor analysis suggested 3 Sullivan statements formed a 'negative personal emotional impact score' (Impact Score, -6 to +6 ). Students with low/ neutral $(-6$ to +3$)$ and high $(+3$ to +6$)$ Impact Score were compared (İ $\$ 2$-tests and ANOVA) and regression analyses undertaken.

Results Respondents were neutral overall (mean Impact Score $=0.3$ ), although with substantial variations. Participants with high Impact Score were likely to be younger, standard course, first-year students, and to have higher depression and distress scores (HADS-D and IRI-Personal-Distress-scale), lower cognitive empathy scores (IRI-Perspective-Taking) and to score more highly on all COLFD sub-scales. Regression analysis showed psychological factors were strongly associated with the Impact Score: COLFD others-dying $(1.29 ; \mathrm{CI}: 1.08$ to 1.50 ; $\mathrm{p}=0.001)$, IRI-Personal-Distress-scale $(0.97$; CI: 0.77 to 1.17 ; $\mathrm{p}=0.001)$, HADS-D $(0.45 ; \mathrm{CI}: 0.17$ to $0.74 ; \mathrm{p}=0.002)$, and
COLFD others-death $(0.31 ; \mathrm{CI}: 0.09$ to $0.54 ; \mathrm{p}=0.006)$ scales were the strongest predictors.

Conclusions Medical students worry about the possible negative personal emotional impact of EOLC when doctors. While medical education may mitigate such concerns, negative expectations appear related to distress, death anxiety and depression. Measures to improve and support psychological well-being may have a positive impact on medical students' attitudes toward EOLC.

\section{REAL TALK - A NOVEL EVIDENCE-BASED, VIDEO-BASED COMMUNICATION SKILLS TRAINING RESOURCE}

R Parry, B Whittaker, M Pino, S Watson, S Hamlyn, C Faull. University of Nottingham, Loughborough University, LOROS Hospice, de Montfort University, Derby University

\subsection{6/bmjspcare-2018-ASPabstracts.21}

Background Much palliative care communication training draws on sparse evidence about practice. Yet training's effectiveness depends on the strength of its underpinning evidence. An empirical, observational science of language and social interaction - 'Conversation Analysis' holds great promise because:

- it is generating copious evidence on communication, and healthcare-communication specifically;

- shows role-played interactions differ from authentic ones in fundamentally important ways;

- recent quantitative evaluations of interventions based on conversation analytic findings have shown effectiveness.

Within a research and training development programme, we designed novel training resources - 'Real Talk' incorporating research findings and clips from video-recorded hospice consultations. We designed Real Talk to complement rather than replace existing resources. We report a preliminary evaluation of Real Talk's strengths and weaknesses.

Method Mixed-methods, qualitative evaluation entailing observations, interviews, and participant-completed feedback questionnaires.

Results We collected data from 11 events, 10 trainers across England, and 150 trainees.

Conclusions Trainees and trainers alike appreciated the video clips and their authentic nature. Observations and reports indicated Real Talk was particularly effective for encouraging participants to both emotionally engage with the nature of palliative care, and actively engage in discussion and overall learning about communication practices. Trainers used the video clips more than they did the research findings components; with a similar pattern seen in most trainees' feedback. Our decision to design Real Talk for trainers to use without initial intensive training meant we could rapidly and widely distribute the resources and evaluate their use. However, this also meant heavy reliance on trainers' existing facilitation skills, and on their allocation of adequate time to familiarise themselves with the materials. We argue that this is also why the research findings-based components were not put to full use by trainers. We are revising Real Talk and its delivery on the basis of our evaluation. 


\section{Free papers 22-24 | Symptom control}

\section{DELIRIUM MANAGEMENT BY SPECIALISTS IN PALLIATIVE MEDICINE: AN ASSOCIATION FOR PALLIATIVE MEDICINE (APM) OF GREAT BRITAIN AND IRELAND SURVEY}

Jason W Boland, Monisha Kabir, Shirley Bush, Juliet Spiller, Miriam J Johnson, Meera Agar, Peter Lawlor. University of Hull, University of Ottawa, Marie Curie Hospice Edinburgh, University of Technology Sydney

\subsection{6/bmjspcare-2018-ASPabstracts.22}

Background Delirium is common in patients receiving palliative care. Management includes assessment, reversal of underlying causes, non-pharmacological interventions and family support. Given recent evidence, antipsychotics should be avoided in mild-moderate delirium.

Methods A 20-question online anonymous survey was emailed to all Association for Palliative Medicine (APM) members. UK and Irish palliative medicine specialists in current clinical practice $(n=859)$ were asked about delirium assessment, management and research priorities.

Results Response rate was 39\% (335/859). Of these, $70 \%$ (234/335) were consultants, $15 \%$ (51/335) were specialist trainee registrars in palliative medicine and $15 \%(50 / 335)$ were associate specialists. Overall, 85\% (285/335) had over 5 years of specialist palliative medicine experience. They worked in a variety, and often multiple settings, across hospital, hospice and community. Delirium guidelines were inconsistently used, with 42\% (115/276) using local guidelines and 38\% (104/276) using no guidelines. Most, 184/314 (59\%) never use a tool to screen for delirium at inpatient admission. Most would use non-pharmacological interventions to manage delirium, either alone $(106 / 275 ; 39 \%)$ or more commonly with an antipsychotic (160/275; 58\%). The majority, 248/273 (91\%) would prescribe an antipsychotic to a delirious patient with distressing hallucinations, with 190/273 (70\%) using typical antipsychotics and 43/273 (16\%) using atypical antipsychotics first line. Most inpatient $(153 / 270 ; 57 \%)$ and community teams $(97 / 161 ; 60 \%)$ do not formally support family carers. Informal verbal support was offered by $24 \%(64 / 270)$ in the inpatient setting and $22 \%(35 / 161)$ in community settings. Research priorities were prevention, prediction of reversibility and management (non-pharmacological and pharmacological).

Conclusion Most responding APM members do not formally screen for delirium at inpatient admission. Many use an antipsychotic along with non-pharmacological interventions to manage delirium, and nearly all prescribe an antipsychotic to treat distressing hallucinations. Further rigorously designed clinical trials are urgently needed in view of management variability, emerging evidence and perceived priorities for research.

\section{THE ASSOCIATION OF BENZODIAZEPINES AND Z-DRUGS WITH MORTALITY IN PATIENTS WITH CANCER: A SYSTEMATIC REVIEW}

Sean B O'Donnell, Martha K Nicholson, Jason W Boland. Hull York Medical School, University of Hull, Hull

\subsection{6/bmjspcare-2018-ASPabstracts.23}

Background Benzodiazepines and Z-drugs are commonly used in patients with cancer for the management of symptoms such as anxiety, agitation and dyspnoea. Clinical staff, patients and relatives have concerns about the impact of these drugs on survival. This potentially decreases prescribing leading to suboptimal symptom control.

The aim of this systematic review was to find and assimilate the evidence assessing the association of benzodiazepines and Z-drugs with survival in patients with cancer, to assist in clinical decision-making regarding the use of these drugs in cancer patients.

Methods Systematic review with narrative synthesis designed and conducted according to the recommendations set out in Preferred Reporting Items for Systematic Reviews and MetaAnalyses-Protocol (PRISMA-P) and PRISMA statements. The review protocol was registered on the PROSPERO prior to commencing the searches. The electronic databases MEDLINE, EMBASE, PsychINFO, Cochrane Library, AMED were searched and hand-searches were performed. Screening, extraction and quality assessment were performed in duplicate.

Results A total of 2257 unique records were identified, 116 full-text articles assessed for eligibility, 18 met the inclusion criteria. These contained data on 4117 patients with cancer. All studies were low or very-low quality. Most studies were conducted in patients in the last days/weeks of life. No study found an association between benzodiazepines and survival in patients with cancer.

Conclusions There is no evidence demonstrating an association between benzodiazepines and survival in patients with cancer. These results should be interpreted with caution as all studies were low/very low quality, most did not report or account for other medications and did not have survival as a primary outcome. No study assessed the effect of long-term benzodiazepines on survival. Therefore, definitive conclusions regarding survival impact of benzodiazepine in patients with cancer can be made. Further investigation using high-quality long-term randomised control trials with survival as a primary endpoint are needed.

\section{THE ASSOCIATION OF ORAL INTAKE WITH THIRST AND SURVIVAL IN ADVANCED CANCER: A SECONDARY ANALYSIS OF OBSERVATIONAL DATA}

Sumandeep Bains, Stephen Mason, Amara Callistus Nwosu. University of Liverpool Medical School, Palliative Care Institute University of Liverpool

10.1136/bmjspcare-2018-ASPabstracts.24

Background Oral fluid intake commonly reduces in advanced cancer patients as they approach the end of their lives. The relationship of oral fluid intake with thirst and survival has not been fully evaluated. Better understanding of how oral fluid intake affects physical health and clinical outcomes may enable health professionals to identify which patients require hydration-based support.

Aim To explore the association of oral fluid intake with thirst and survival in advanced cancer patients.

Method A secondary analysis of an observational study database of 90 patients with advanced cancer from a previous study by Nwosu et al 2016. Regression analysis evaluated the relationship between oral fluid intake and thirst, and examined the predictive properties of recorded variables. Kaplan-Meier analysis evaluated the relationship between survival and oral fluid intake. A Cox regression was used to adjust for potential confounding factors: performance status, metastatic disease and age. 